Received June 23; revised October 29, 1960

${ }^{1}$ Wilkinson, R. T., in Progress in Clinical Psychology (Grune and Stratton, New York, 1969).

${ }^{2}$ Baddeley, A. D., Psychon. Sci., 10, 341 (1968).

${ }^{3}$ Legge, D., Brit. J. Psychol., 56, 243 (1965).

- Legge, D., Steinberg, H., and Summerfield, A., Percep. Mot. Skills, 18, 549 (1964).

${ }^{5}$ Brown, I. D., Ann. Occup. Hyg., 5, 131 (1962).

\section{Transmission of Plasmodium berghei (NK65 Strain) by Anopheles annulipes Walker}

SINCE the isolation of Plasmodium berghei from the treo rat Thamnomys surdaster in the Congo ${ }^{1}$, there have been many attempts to infect mosquitoes in the laboratory and establish cyclical transmission. This has been necessary because the natural vector Anopheles dureni can be colonized only with difficulty ${ }^{2}$.

Successful infection of $A$. stephensi, $A$. gambiae, $A$. quadrimaculatus and $A$. maculipennis var. atroparvus was first achieved by Yoeli and $W_{a l l}^{3}$ and of $A$. quadrimaculatus and $A$. aztecus by Perez-Reyes 4 , but sporozoites reached the salivary glands in only a small proportion of infected mosquitoes, and these were low density infections. Transmission to animals by these and subsequent workers resulted from inoculation of sporozoites obtained from oocysts and salivary glands. In 1965 Yoeli, Most and Bones found that the sporogonic cycle could be completed successfully if the mosquitoes were kept at $21^{\circ}-22^{\circ} \mathrm{C}$ during and after the infective feed. In these conditions 42.5 per cent of $A$. quadrimaculatus developed oocysts and 75 per cent of these had salivary gland infections, but only 100-300 sporozoites were found in the infected glands. In $A$. stephensi, 50.5 per cent had stomach infections and 95 per cent of these mosquitoes had heavy salivary gland infections, comparable with those found in naturally infected $A$. dureni. Even in these optimum environmental conditions, however, $A$. albimanus was a very poor experimental vector, with a stomach infection rate of only 3 per cent.

We have recently made the first successful attempt to incriminate Anopheles (Myzomyia) annulipes Walker, 1856, as an experimental vector of $P$. berghei using an anopheline from the Australasian zoogeographical region. The mosquitoes used came from a laboratory colony maintained by induced copulation and were originally collected from the environs of Sydney. A gametocyteproducing strain of $P$. berghei (NK65), maintained by cyclical transmission in the laboratory of Professor Meir Yoeli in New York, was taken to Sydney by air in white mice in March 1969, and then maintained in white mice in our laboratory by intraperitoneal inoculation of infected blood. Mosquitoes were fed on infected mice between the second and ninth day after inoculation, when the gametocyte rate was high, after which time they were maintained in a humid atmosphere at $20^{\circ}-21^{\circ} \mathrm{C}$. These mosquitoes were allowed to feed on 21-30 day old albino rats, 13 days and more after the infective meal. Dead and dying mosquitoes were dissected daily and examined for sporozoites and oocysts.

We were successful in May 1969, using the initial strain of parasite which had been stored in liquid air since March 1969. During 6 weeks, 145 mosquitoes which had been allowed to feed on infected mice were dissected and, of these, sixty-seven $(46.2$ per cent) had stomach infections of from one to more than 150 oocysts. Salivary glands were examined in only a small proportion of theso mosquitoes, for most were desiccated or decomposed when dissected. Sporozoites were seen in only four mosquitoes, all with a heavy infection.

Transmission of $P$. berghei by $A$. annulipes was obtained when five rats challenged by bites of mosquitoes previously fed on mice became infected. But no infection developed in rats bitten by mosquitoes which had taken an infective blood meal less than fifteen days previously. The course of infection in the infected rats was as follows. In the first rat, challenged by six or seven mosquitoes, 15 and 16 days after the infective meal, parasites were observed on the fourth day, and an increasing parasitaemia developed until the animal died, with severe anaemia, on the twentieth day. Almost all of the red cells were heavily parasitized, and many of the nucleated red cells present were also infected. Yoeli (personal communication) commented that these parasites had very similar morphology to those described as his N.Y.U. ${ }_{2}$ strain $^{6}$.

Parasites appeared in the second rat 6 days after it had been bitten by four mosquitoes fed 16 days previously. An increasingly high parasitaemia developed, and the rat. died on the twenty-seventh day. Parasites appeared in the third rat 7 days after it had been bitten by a single mosquito, fed 15 days before. This rat died on the twentieth day with a high parasitaemia.

In the two other rats the parasitaemia spontaneously disappeared. In the first case three mosquitoes which had fed 20 days previously were used to challenge the animal. A few parasites were present in the peripheral blood after 5 days but could not be demonstrated on the sixth or subsequent days. Blood was subinoculated intraperitoneally into three white mice on the seventh day, but they did not become infected. In the other rat, infected by four mosquitoes which had fed 16 days earlier, parasitaemia was observed on the fifth day and a high parasitaemia developed when, on the twenty-third day, there were more parasites present than red blood. cells. On the twenty-fifth and subsequent days, no parasites could be demonstrated.

We consider these results to be important because mice but not hamsters were available as the gametocyte carrier and because another anopheline species, not related to the natural vector, has been demonstrated as an experimental vector of rodent malaria.

We thank Professor Yoeli for the strain of $P$. berghei and for his encouragement. We also thank Privates Gulley and Green and Corporal Kreutzmann of the 1 Malaria Research Laboratory, RAAMC, and $\mathrm{Mr}$ J. C. Walker of the School of Public Health and Tropical Medicine for technical assistance.

\section{Elizabeth C. KaluCy}

1 Malaria Research Laboratory, RAAMC,

School of Public Health and Tropical Medicine,

University of Sydney.

\section{B. McMiluan}

School of Public Health and Tropical Medicine,

University of Sydney.

Received September 15, 1969.

${ }^{1}$ Vincke, I. H., and Lips, H., Ann. Soc. Belge Med. Trop., 28, 97 (1948).

2 Yoeli, M., and Boné, G., Riv. Malaria, 46, 1 (1967).

3 Yoeli, M. and Wall, W., Nature, 168, 1078 (1951).

4 Perez-Reyes, R., J. Parasitol., 39, 603 (1953).

Yoeli, M., Most, H., and Boné, G., Ann. Soc. Belge Med. Trop., 45, 267 (1965).

${ }^{8}$ Yoeli, M., and Most, H., Trans. Roy. Soc. Trop. Med. Hyg., 54, 549 (1960).

\section{Hollow Casts of Lungs for Experimental Purposes}

Severat techniques have been described for producing solid casts of human and animal respiratory tracts in wax ${ }^{1}$, Wood's metal' and 'Vinylite's. Made with care, these casts keep the fine detail of the airways extending as far as the respiratory bronchioles. But because the casts are "negatives", in that they represent the airspaces rather than the airway walls, their use has been limited to demonstrations of anatomical features and pathological changes ${ }^{4}$. Hollow positive casts for use in airflow studies. have been made from these negatives by conventional casting techniques; some made in resin ${ }^{5,6}$ have contained. 